\title{
Accretion signatures in massive young stellar objects
}

\author{
Robert D. Blum \\ Cerro Tololo Interamerican Observatory, Casilla 603, La Serena, Chile \\ email: rblumctio.noao.edu
}

\begin{abstract}
I review recent observational progress on the search for accretion signatures in massive young stellar objects (MYSOs). In this context, the primary tool will be $1-5 \mu \mathrm{m}$ high resolution $(\lambda / \Delta \lambda=50,000)$ spectroscopy. The observational regime is the phase between massive star formation (collapse, accretion, mergers, etc) and the fully revealed OB star photosphere, the phase for which the term "Massive Star Birth" was originally coined. In this phase, we seek both the signature of the earlier accretion process and the photospheric markers of the truly massive star which is on the main sequence. The sample of objects used in this endeavor are massive young stars located in nascent clusters powering Galactic giant H II regions. Emission in the $2.3 \mu \mathrm{m} \mathrm{2-0} \mathrm{vibrational-rotational} \mathrm{bandhead} \mathrm{of} \mathrm{CO}$ is observed as are the ionized lines of Hydrogen, Helium, and other abundant elements. High spectral resolution is key in giving geometrical clues to the circumstellar emission sources, both through individual line profiles (i.e. shapes) and positions.
\end{abstract}

Keywords. stars: early-type, stars: formation, stars: fundamental parameters

\section{Introduction}

One of the main goals of this meeting is review the progress on the understanding, both observationally and theoretically, of the formation process(es) of massive stars. Even the most basic notions of massive star formation are not yet fully defined/confirmed by detailed observations. In particular, the relative importance of accretion vs merger processes will be discussed at length by many authors. In the present contribution, I will review recent progress on this topic from the point of view of near infrared spectroscopic observations (i.e observations in the approximate range $1-5 \mu \mathrm{m}$ ).

Though the near infrared regime allows to penetrate much deeper into or through dusty environments, the situation with massive stars still presents a significant challenge. This is because the time scale for formation must be rapid and so average accretion rates are high leading to a central object which is necessarily heavily obscured by overlying, infalling material. Even collisional processes must be relatively short, and, it is imagined, would produce a significant column of obscuring circumstellar material during the initial phases. Thus, near infrared techniques are complementary to other longer wavelength approaches which will be more effective in observing the earliest phases of massive star formation. There are also distinct advantages to near infrared observations. New generation $8 \mathrm{~m}$ class telescopes equipped with high spectral and angular resolution instruments allow sensitive observations over a much larger distance range than was previously possible. Thus the volume which can be searched is much greater, and significant samples of these rare objects can be identified.

In the present context, a massive young stellar object (MYSO), is taken to be the object observed just before a normal OB star is revealed emerging from its natal birth environment. This phase follows the earlier phases outlined by Churchwell (2002) but 


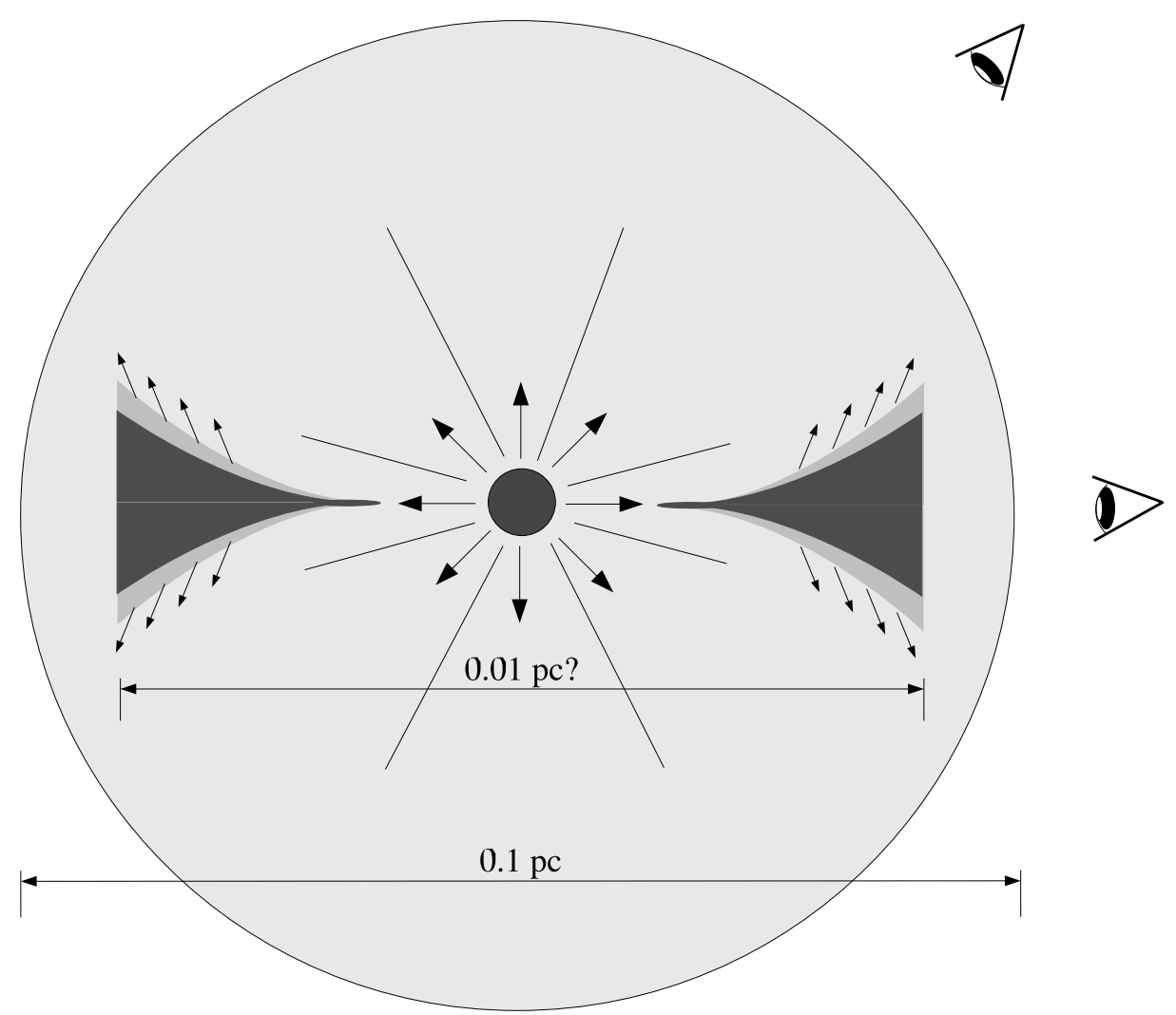

Figure 1. Cartoon of a massive young stellar object (MYSO) as it might appear in the late stages of the star birth phase, just before becoming a revealed OB star.

can naturally include the ultra compact HII region (UCHII) phase in which the hot star is ionizing its immediate surroundings. With high spectral and angular resolution data, remnant accretion disks can be identified through their kinematic signatures (if they exist). In an ideal case, one catches the MYSO just at the proper time such that the disk is still observed while the OB star photospheric features are revealed in the near infrared (e.g. in the $K$-band). This latter identification is critical to obtaining key information on the nature of the central star which otherwise has to be inferred from other data (typically a "luminosity" derived mass from long wavelength baseline spectral energy distributions).

With these points in mind, an MYSO is sketched schematically in Figure 1. The principle components are a central source (a bonafide star on the ZAMS, though perhaps not at its final mass), ionizing flux and a stellar wind which are helping to clear away the birth material and allow the photosphere to be seen, an inner disk, and a more extended disk/torus with a possible disk wind. Everything is surrounded by the HII region created as the ionizing source acts on the molecular cloud surrounding the nascent star. Obviously, the scales will evolve. The outer scale in this cartoon is consistent with radio observations of UCHII regions and with near infrared observations of MYSOs in massive star clusters. The inner scale is approximately an upper limit for a fairly evolved MYSO. It corresponds to the observed torus structure seen in the source NGC3576 \#48 (Blum et al. 2004). Also as indicated in the figure, the observed properties of any particular 
MYSO will depend on the viewing angle of the observer. Barbosa et al. (2003) find 10 and $20 \mu \mathrm{m}$ spectral energy distributions of different MYSOs are consistent with these objects being seen through different lines of site through the circumstellar material in a model similar to that shown in Figure 1. Blum et al. (2004) further present evidence that the detailed line profiles from presumed stellar wind components of MYSOs show behavior consistent with the model of Figure 1 seen at different orientation.

One might add a molecular outflow along the disk/torus axis. It is not clear whether this component has already turned off for the MYSOs discussed here. This is likely the case for any MYSO which has photospheric features observed, but younger objects may still show evidence of out flows, for example, in the molecular Hydrogen line at $2.12 \mu \mathrm{m}$. Ishii et al. (2001) presented spectra for IRAS selected precursor-Herbig AeBe stars. The most massive of these would be considered MYSOs in the present context. These authors found that molecular hydrogen emission is typical of only the most obscured "class I" type objects in their sample. This is also the case for our own unpublished high resolution spectra for MYSOs in NGC3576. The most buried of the sources in Barbosa et al. (2003) shows evidence of molecular out flow in the $2.12 \mu \mathrm{m}$ line (blue shifted line emission up to $\approx 100 \mathrm{~km} \mathrm{~s}^{-1}$ ).

\section{Searching for MYSOs}

One of the earliest investigations of $\mathrm{CO}$ overtone emission in circumstellar environments was by Scoville et al. (1983) who observed the Becklin Neugebauer (BN) object at high resolution. BN is, of course, an MYSO (in the nearby star forming region of Orion), consistent with spectral type approximately B1. These observations were made possible with an FTS spectrograph owing to BN's close proximity and hence brightness. Today's modern grating spectrometers and large telescopes allow us to return to the subject because they allow much fainter objects to be observed. Larger samples of stars are now being observed, and we can begin to make some conclusions, though many issues, even basic ones remain.

Recent searches have turned up MYSOs primarily through the larger effort to find and characterize the stellar content in giant HII (GHII) regions. See, for example, Hanson et al. (1997), Conti \& Blum (2000), Blum et al. (2001), Figuerêdo et al. (2002), Alves \& Homeier (2003), and Bik (2004). The thesis by Bik is a nice reference to the subject of recent work in this field as well as a source of new work in itself. The exploration of GHII regions is covered further in these proceedings (see contributions by A. Damineli \& J. Alves).

Figure 2 shows a representative near infrared spectrum of a MYSO from the GHII region W49A. The rising red continuum, Brackett emission lines (note the wonderful transparency in the region near $1.9 \mu \mathrm{m}$ for this spectrum taken at UKIRT on Mauna Kea), He I, and FeII emission. The latter is common (see Ishii et al. 2001, Blum et al. 2001, Bik 2004), but does not appear in all near infrared spectra. Other lines which have been observed are molecular Hydrogen, $\mathrm{Na}$, and CO (modeling the CO line profiles using high dispersion spectra is discussed below). In some cases, the circumstellar obscuration is low enough that photospheric lines can be seen, but this is more rare as discussed below (see also Hanson et al. 1997 and Figuerêdo et al. 2005).

MYSOs typically stand out in near infrared color magnitude diagrams. In W49A and W31, for example, two of the brightest $K$-band objects are MYSOs. Of course, most of this luminosity is reprocessed. This leads to the problem that near infrared magnitudes can not easily be used in the case of MYSOs to infer anything about the fundamental properties of the central star. Hillenbrand et al. (1992) calculated models of flat disks 


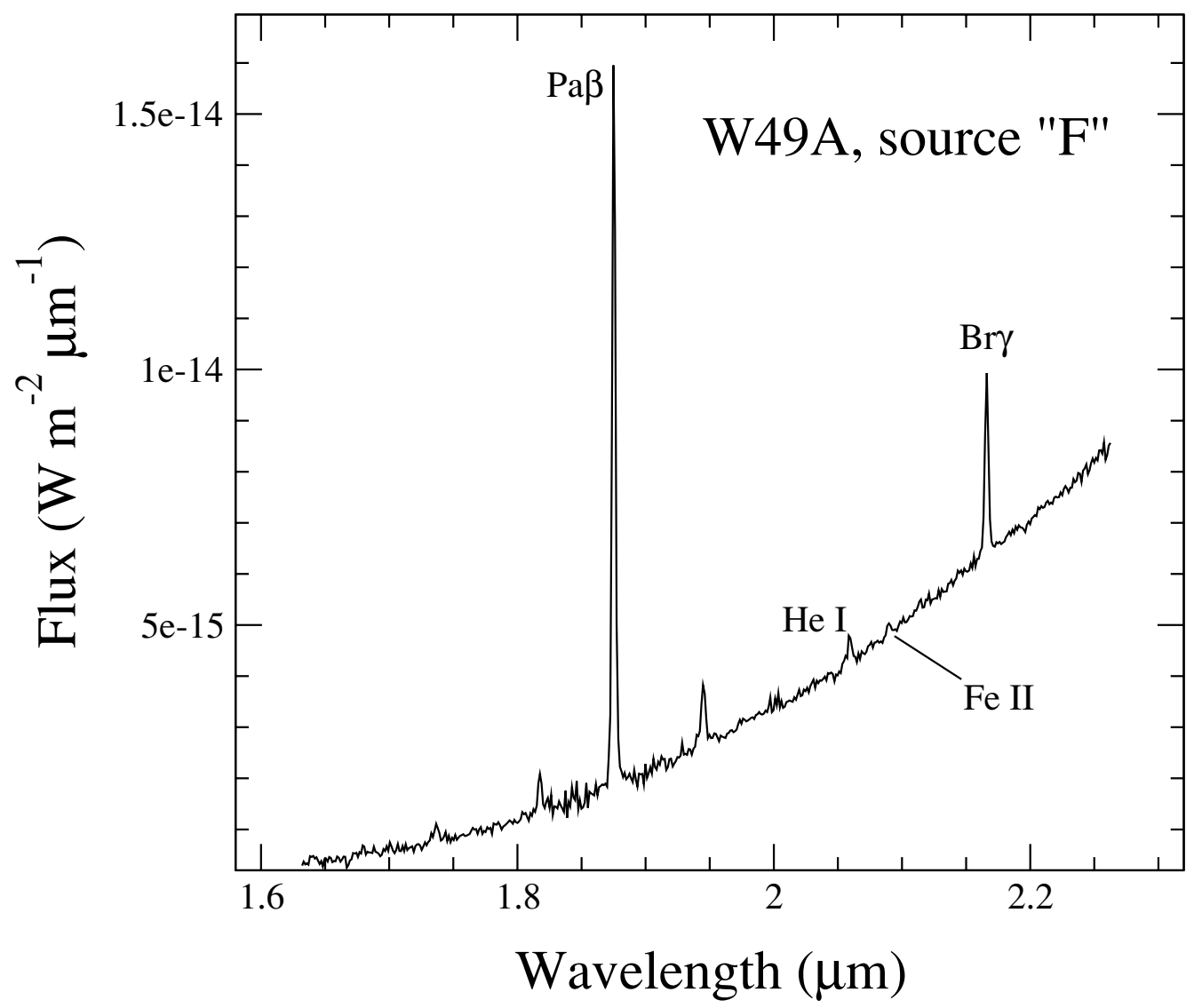

Figure 2. Low resolution $H$ and $K$-band spectrum of a typical massive young stellar object (MYSO), in this case, the luminous UCHII source W49F (see De Pree et al. 2000). This UKIRT CGS4 spectrum was kindly provided by Tom Geballe.

which reprocess light from the central star. For a face on disk, an O7 star can have an excess luminosity of four magnitudes! Excesses at $H$ and $J$ can be nearly as large, and this can lead to serious errors in calculating extinction values if one or both of these bands is assumed to have a negligible excess.

\section{CO Overtone Emission as an Accretion Tracer}

The history of this work is based on the work developed for low mass YSOs. The most important aspect for the present discussion is the general success of the accretion disk model for YSOs coupled with the straightforward observation and modeling of kinematic tracers such as the $\mathrm{CO}$ first overtone vibrational-rotational transitions which result in bandheads near $2.3 \mu \mathrm{m}$ at the red end of the $K$-band. CO emission requires dense $\left(\lesssim 10^{1} 0 \mathrm{~cm}^{-3}\right)$ gas as is expected in an accretion disk. Such densities allow the molecule to self-shield, and so it can survive in the harsh radiation field of massive stars. The modeling of CO profiles follows the approach used by Carr (1993), Chandler et al. (1995), Najita et al. (1996), and Kraus et al. (2000). The complexity of the analysis varies, but the main point is the same: the observed profiles for the CO bandheads can be well fit by geometrically thin disks with Keplerian rotation. This does not necessarily rule out 


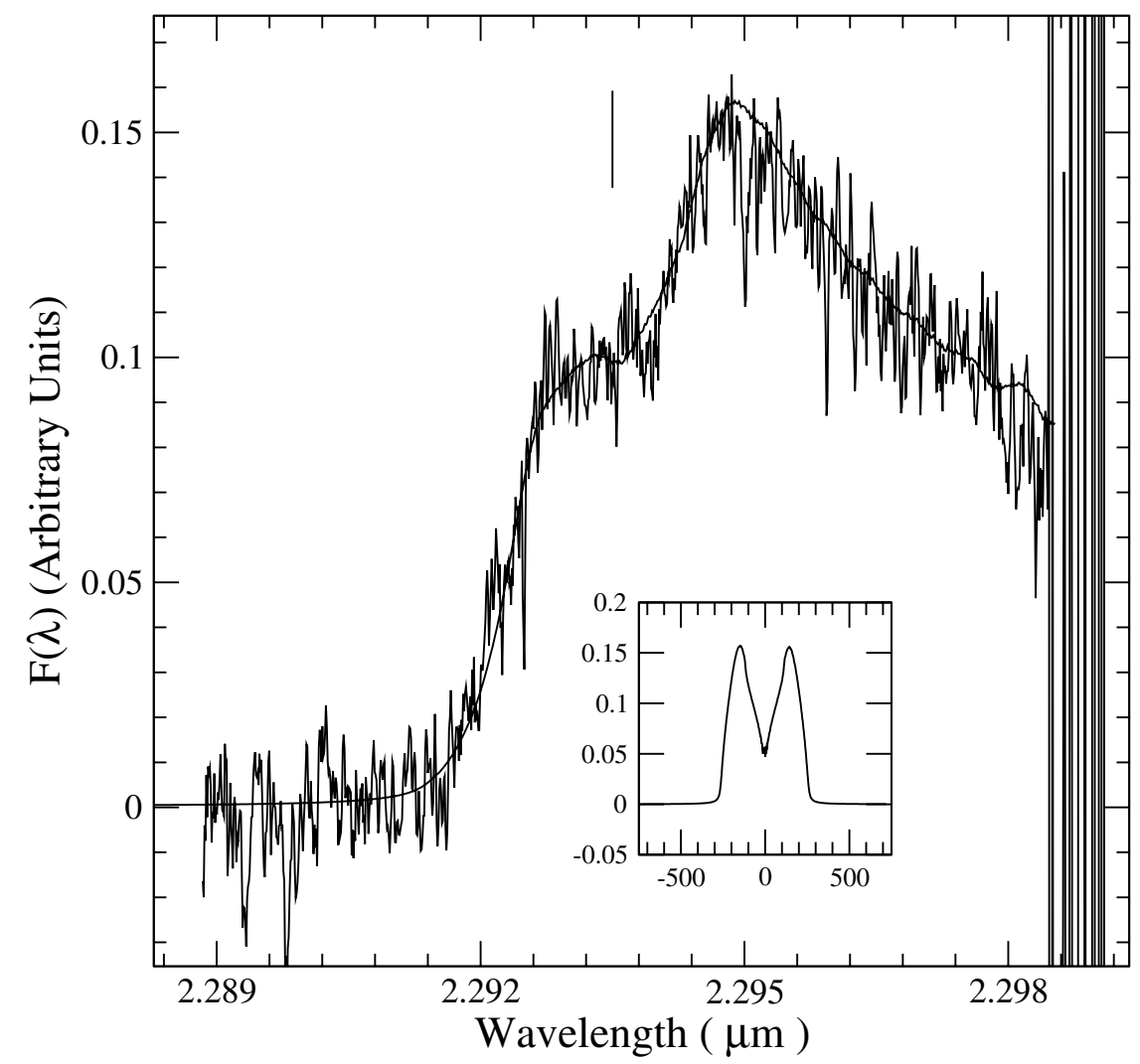

Figure 3. Gemini South-Phoenix spectrum of the $2.3 \mu \mathrm{m}$ CO bandhead emission from source M17-268. The short vertical line marks the vacuum rest wavelength of the band head, and the inset shows the emission-line profile for a single line in the band head. See Blum et al. (2004) for details.

other emission mechanisms (Chandler et al. 1995), but generally, the disk provides the best fit and simplest explanation.

Two recent studies (Bik \& Thi 2004 and Blum et al. 2004) apply similar techniques to samples of MYSOs (the source $11097 \mathrm{nr} 1218$ of Bik and Thi was observed by Blum et al. and identified as NGC3576 \#48 by those authors). Both groups conclude that the CO profiles observed at high spectral resolution $(R=10000$ and 50000 for Bik and Thi and Blum et al., respectively) are generally well fit by Keplerian disks. The derived disk parameters are similar and indicate $\mathrm{CO}$ emission zones within $1 \mathrm{AU}$ of the central sources. An example taken from Blum et al. (2004) is shown in Figure 3. The continuum subtracted profile is fit by a disk with vsini at the inner radius of $260 \mathrm{~km} \mathrm{~s}^{-1}$. See Blum et al. (2004) for details.

An issue which should be settled as more stars with CO emission are observed at high resolution is that most of the stars observed to date have relatively narrow profiles. Any given star could be seen at a relatively high inclination (more face on), but we should expect to see similar numbers of high velocity profiles as well (such as in Figure 3). If the $\mathrm{CO}$ emitting region is typically partially obscured by the geometry of the circumstellar disk, then the observations might be biased toward narrow profiles. A particular edgeon geometry that could produce a narrow profile was suggested for MCW349 by Kraus 


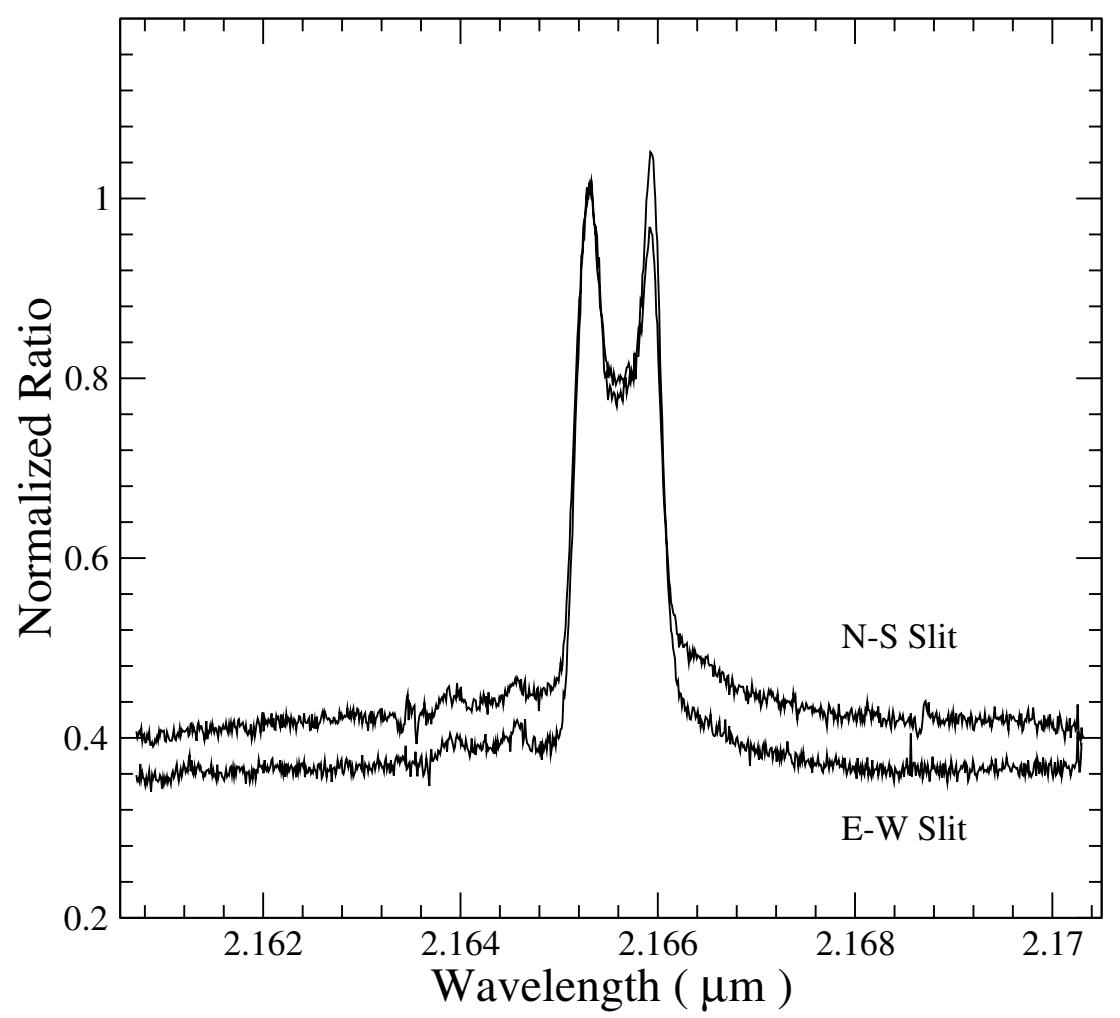

Figure 4. Gemini South-Phoenix Br $\gamma$ spectrum of the source \#48 in NGC3576. Blum et al. (2004) interpret this double peaked emission as possibly arising in a torus.

et al. (2000). In general, such a bias means that the CO profiles, while still valuable to demonstrate rotation) would be less useful in deriving actual disk properties.

\section{Lines from Ionized Material}

The emission lines from recombination of ionized Hydrogen and Helium are obviously of great interest to the study of massive stars in their natal environments.

Figure 4 shows a spectrum of the MYSO \#48 (Blum et al. 2004, see Bik \& Thi 2004 as well). The double peaked emission is interpreted as arising in a torus or expanding shell. The CO emission in this source has a mean velocity distinct from the emission shown in the Figure. This suggests that the two do not arise from combined disk/torus as depicted in Figure 1. The lower resolution spectrum presented by Bik (2004) does not show this double peak, though the slit width and velocity splitting $\left(95 \mathrm{~km} \mathrm{~s}^{-1}\right)$ should have allowed Bik to see it. The full 2-D image presented by Blum et al. (2004) shows a rich and complex spatial/kinematic morphology which is broadly consistent with the cartoon MYSO shown in Figure 1. Weak He I emission is seen in this figure as the two "bumps" to the blue of the Br emission. The He I emission follows the same general morphology as that for $\operatorname{Br} \gamma$ in the full 2-D image.

High dispersion spectra are needed to clearly distinguish multiple physical components in MYSOs. A primary component is the ionized "disk wind" as indicated by the small arrows in Figure 1. This wind arises from the ionized surface of the circumstellar disk. It carries the rotation signature of the disk zone from which is arises as the gas is heated 


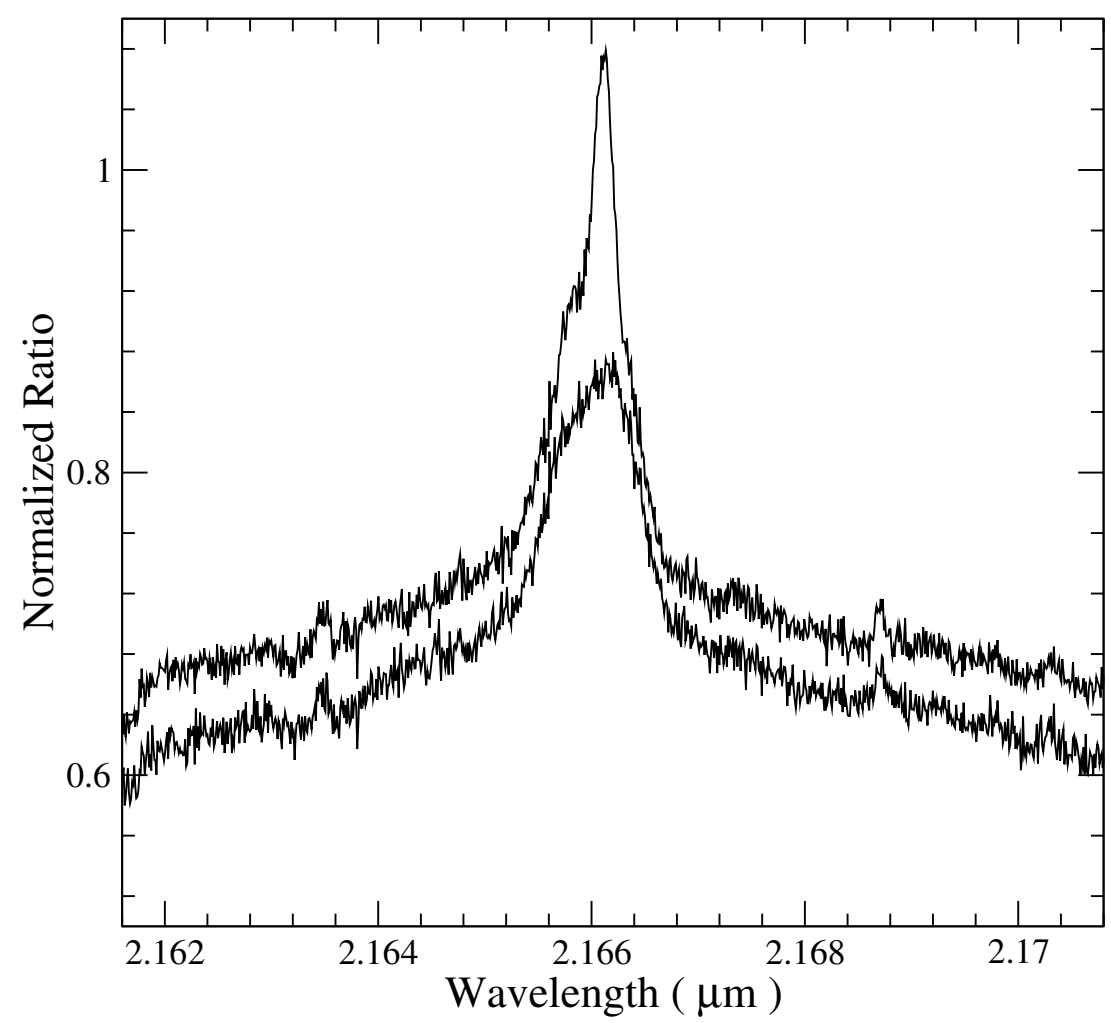

Figure 5. Same as Figure 4, but for the source 275 in M17. Note the broad wings which are interpreted as coming from a possible stellar wind while the central component of emission is associated with the disk in this source seen at a high inclination angle (more face on, as seen in the $\mathrm{CO}$ emission too; see Blum et al. 2004 for details).

and removed from the disk surface. A second component is the stellar wind itself. This radiatively accelerated wind should be relative fast compared to the disk wind. These features of the model MYSO are consistent with the spectrum of M17-275 shown in Figure 5. The "stellar wind" component has a full extent of at least $\pm 300 \mathrm{~km} \mathrm{~s}^{-1}$.

\section{Accretion in Massive Young Stars}

The preliminary studies mentioned in the preceding sections indicate that massive stars form by accretion. Yet, by their nature, these objects are heavily obscured and their central masses must be estimated by secondary means. Usually this means some type of luminosity measurement is converted into a mass based on stellar models. Ideally, we would like more secure mass estimates (e.g. photospheric spectra) in order to more accurately assess disks and their relevant time scales with respect to stellar mass.

Do all massive stars form by accretion, or is there a cut-off above which collisional processes contribute or take over? The preliminary work suggests at least the second rank, i.e. early B, late O, stars show compelling evidence of remnant accretion disks in their near infrared spectra. But what about more massive O stars? Here, nature is working against us again. The more massive stars will naturally have shorter life times, and the action of their stronger winds and harsher UV radiation will help destroy their disks more rapidly. 
An approximate tally of objects which show $\mathrm{CO}$ emission in the $K$-band from Chandler et al. (1995), Ishii et al. (2001), Bik \& Thi (2004), and Blum et al. (2004) results in some $\sim 25$ sources (perhaps $1 / 3$ have high resolution spectra). These are late $\mathrm{O}$ stars at the most massive, but most are B type and of these the lower masses (or at least luminosities) predominate. Further, Fuente et al. (2003) show through mm observations that Herbig Be stars have less massive remnant disks than similar A stars. The distribution of "disk" like OB stars is thus consistent with higher mass objects being more efficient at dissipating their circumstellar disks. Observations in GHII region star clusters are also consistent with this idea. In W31, Blum et al. (2001) find a MYSO (the most luminous object in the $K$-band) is likely an early B star while four mid O type stars in the same cluster show no spectroscopic tracers of circumstellar disks in the near infrared.

Dissipation times scales are expected to be inversely related to stellar mass on the upper main sequence; see the recent review by Hollenbach et al. (2000) and the contribution to these proceedings by Lizano. Richling \& Yorke (1997) produced detailed numerical models for B stars of a range of masses and mass loss properties showing that such stars should disperse their natal disks within $10^{5}$ and a few by $10^{6}$ years. Clearly, finding accretion signatures around more massive stars with the present techniques will be very difficult. All the relevant timescales conspire to make such objects rare. To date, no signatures have been found in MYSOs earlier than late O or early B type. In the oral version of this contribution, I presented evidence of a mid-type O star (W51-2902) which my collaborators and I thought exhibited the composite spectrum of an $\mathrm{O}$ star and $\mathrm{CO}$ emission. The $\mathrm{CO}$ component of this spectrum was not real, but was due to the absorption contribution of a close companion ( $\mathrm{G}$ type) to the A0V star used to correct for telluric absorption in W51-2902. The error was entirely ours, though we had not known the A0V telluric standard which was observed in Q mode at Gemini North was actually a binary with a $\mathrm{G}$ type companion. Such stars have weak $\mathrm{CO}$ absorption which is not readily apparent among the strong telluric absorptions near the same wavelengths.

\section{Acknowledgements}

I would like to thank my collaborators, Peter Conti, Augusto Damineli, Lys Figuerêdo, and Cassio Barbosa for their many contributions to our joint study of massive stars in their birth environments. It has been a very productive and enjoyable endeavor for me.

Some of the data presented here are based on observations obtained at the Gemini Observatory, which is operated by the Association of Universities for Research in Astronomy, Inc., under a cooperative agreement with the NSF on behalf of the Gemini partnership: the National Science Foundation (United States), the Particle Physics and Astronomy Research Council (United Kingdom), the National Research Council (Canada), CONICYT (Chile), the Australian Research Council (Australia), CNPq (Brazil) and CONICET (Argentina).

\section{References}

Alves, J. \& Homeier, N. 2003, ApJ, 589, L45

Barbosa, C. L., Damineli, A., Blum, R. D., \& Conti, P. S. 2003, AJ, 126, 2411

Bik, A. 2004, PhD Thesis, Astronomical Institute Anton Pannekoek, the Netherlands

Bik, A. \& Thi, W. F. 2004, A\&A, 427, L13

Blum, R. D., Barbosa, C. L., Damineli, A., Conti, P. S., \& Ridgway, S. 2004, ApJ, 617, 1167

Carr, J. S., Tokunaga, A. T., Najita, J., Shu, F. H., \& Glassgold, A. E. 1993, ApJ, 411, L37

Chandler, C. J., Carlstrom, J. E., \& Scoville, N. Z. 1995, ApJ, 466, 793 
Churchwell, E. 2002, ARAA, 40, 27

De Pree, C. G., Wilner, D. J., Goss, W. M., Welch, W. J., \& McGrath, E. 2000, ApJ, 540, 308

Figuerêdo, E., Blum, R. D., Damineli, A., \& Conti, P. S. 2002, AJ, 124, 2739

Fuente, A., Rodríguez-Franco, A., Testi, L., Natta, A., Bachiller, R., \& Neri, R. 2003, ApJ, 598, L39

Hillenbrand, L. A., Strom, S. E., Vrba, F. J., \& Keene, J. 1992, ApJ, 397, 613

Hollenbach, D. J., Yorke, H. W., \& Johnstone, D. 2000, Protostars and Planets IV, 401

Ishii, M., Nagata, T., Sato, S., Yao, Y., Jiang, Z., \& Nakaya, H. 2001, AJ, 121, 3191

Kraus, M., Krügel, E., Thum, C., \& Geballe, T. R. 2000, A\&A, 362, 158

Najita, J., Carr, J. S., Glassgold, A. E., Shu, F. H., \& Tokunaga, A. T. 1996, ApJ, 462, 919

Richling, S. \& Yorke, H. W. 1997, A\&A, 327, 317

Scoville, N., Kleinmann, S. G., Hall, D. N. B., \& Ridgway, S. T. 1983, ApJ, 275, 201 